\title{
Inter hemispheric connectivity and attention in patients with disorders of consciousness after severe traumatic brain injury
}

\begin{abstract}
The study of the function of attention in the dynamics of disturbed and recovering consciousness in severe cerebral pathology is becoming more relevant-due to the increasing number of patients with prolonged disorders of consciousness and significant difficulties in their rehabilitation.

Objective: to determine the electroencephalographic correlates of modality-nonspecific attention using a clinical model of severe traumatic brain injury (STBI).

Patients and methods: 35 patients with STBI in the dynamics of post coma recovery of mental activity (a study group) and 23 healthy subjects (a control group) were examined. Changes in the pattern of EEG and in the indices of its coherence in the presence and activation of different forms of attention (an orienting response to the sound and eye opening; involuntary and voluntary visual forms), by applying specially developed computerized techniques, were investigated. The features of associated with attention changes in interhemispheric EEG coherence (EEGInterCoh) with the data of $3 \mathrm{~T}$ diffusion tensor tractography of the corpus callosum (CC) were compared.

Results: Attention disorders were shown to be essential and an «axial disorder» in patients with SBI. There were statistically confirmed qualitative and quantitative differences attention-associated changes in the EEG pattern and inter hemispheric coherence in reversible and chronic unconsciousness. The important favorable prognostic sign proved to be reactive changes in inter hemispheric EEG relations, including frontal ones characterized by the absence of clear external manifestations of consciousness in the very earliest stages. There was a significant correlation between the preservation of $\mathrm{CC}$ tracts (primarily, the rostrum, anterior portion, and splenium) and attention- related reactivity of EEG Inter Coh, which reflects the specific, though nonrigid, structural determinacy of the latter.
\end{abstract}

Keywords: attention, posttraumatic unconsciousness, electroencephalography
Volume 8 Issue 4 - 2018

\section{Sharova EV,' Pogosbekyan EL,', Korobkova EV,' Zaitsev OS, ${ }^{2}$ Zakharova NE, ${ }^{2}$ Chelyapina MV,' Fadeeva LM, ${ }^{2}$ Oknina LB,' Pronin IN, ${ }^{2}$ Potapov $\mathrm{AA}^{2}$ \\ 'Institute of Higher Nervous Activity and Neurophysiology of RAS, Russia \\ ${ }^{2}$ Federal State Autonomous Institution, N.N. Burdenko National Scientific and Practical, Center for Neurosurgery of the Ministry of Healthcare of the Russian Federation, Russia}

Correspondence: Sharova EV, Institute of Higher Nervous Activity and Neurophysiology of RAS, Russia, Email ESharova@nsi.ru

Received: June 22, 2018 | Published: August 17, 2018

\section{Introduction}

Attention is one of the basic components of consciousness, ${ }^{1-3}$ thus studying the dynamics of its manifestations in disordered and recovering consciousness is becoming more relevant due to an increasing number of patients with depressed consciousness and serious rehabilitation difficulties. ${ }^{4-6}$ The external signs of attention (involuntary eye movements, eye tracking and fixation) are what is considered as first important indicators of waking up from a coma, emerging and restoring consciousness, ${ }^{7,8}$ largely determining the success of consciousness recovery in patient with cerebral pathology. ${ }^{9,10}$ In case of a severe traumatic brain injury (STBI), attention disorders are shown to play a key role "axial disorder", whose degree of regression enables us to evaluate the dynamics of a patient's condition and rehabilitation effectiveness. ${ }^{11}$

At the same time, many issues concerning attention (AT) in case of depressed consciousness have not been sufficiently studied. Further research is required on a morphofunctional substrate providing different types of modallty-nonspecific attention in disorders of consciousness (DOC). The current understanding of the connection of involuntary attention with the state of the regulatory brainstem structures (medulla oblongata and midbrain) and that of voluntary/ purposeful attention with the state of the basal ganglia and medial temporal lobe, ${ }^{12}$ is being redefined and completed with new data on the found hemispheric systems of modality-nonspecific attention (frontoparietal and cingulo-opercular), ${ }^{13,14}$ and, in case of voluntary, selective attention, with a scheme of selective cortical activity modulation by the fronto-thalamic regulatory system, including the mediodorsal thalamic nucleus and the prefrontal cortex as a "core". ${ }^{15}$ There are also premotor theories of attention, ${ }^{16,17}$ suggesting that eye movements and attentional shifts are provided by closely related mechanisms involving, besides the premotor cortex, the nuclei colliculi superioris and the posterior parietal cortex.

Among attention-related electrographic markers, the method of long-latency evoked potentials is widely used. ${ }^{18-21}$ The other conventional method of electroencephalography (EEG), with which the electrophysiology of attention ${ }^{22-23}$ started, is far from exhausted. The indicators of different types of attention include an increase in EEG local spatial synchronization ${ }^{12,24}$ and EEG high-frequency $(30-170 \mathrm{~Hz})$ activity, ${ }^{25,26}$ specific behavior of certain individual rhythms, ${ }^{27,28}$ as well as the attention-related features of topography of changes in EEG, which reflect the participation of various activating brain subsystems. ${ }^{29}$

Earlier, we suggested a methodological algorithm for EEG studies of modality-nonspecific human AT (orienting response, voluntary and involuntary attention) and identified a number of corresponding EEG markers in healthy adult subjects. ${ }^{30}$ Those include the topography of 
changes in the intra- and especially interhemispheric coherence of EEG (EEGInterCoh): unidirectional reactivity of the fronto-polar and anterolateral temporal connectivity in the context of frontothalamic activation; reciprocal, in case of orienting response in the context of fronto-thalamic and hippocampal. ${ }^{15,31}$ Concurrently with diffusely decreasing coherence, we found its local increase in the representation area of an active sensor, which coincides with the area of an increased oxygenation of the blood, according to fMRI. ${ }^{30}$ The present work aimed to study various EEG correlates of modality nonspecific attention in the dynamics of mental recovery from coma to clear consciousness in the clinical framework of STBI.

Given the significance of reactivity of interhemispheric coherence as an attention marker found in the norm ${ }^{30}$ a special interest was taken in the comparison of EEGInterCoh with the state of the corpus callosum tracts, which anatomically provide interhemispheric connectivity. It was also suggested by the recent data on the significant correlation of the degree of the corpus callosum damage with the severity of the condition, the degree of consciousness depression in patients with STBI and its outcome. ${ }^{32}$

\section{Methods}

The test group consisted of 35 patients with STBI and concurrent depression of consciousness, undergoing mental recovery. The patients were aged 8 to 60 (with average of 28). According to the complex clinical examination (neurological, psychiatric, computed tomography, MRI), all the patients had multiple brain damage of different types: contusions with edema, intracranial hematomas with dislocation, concurrently with diffuse-axonal brain damage. The current clinical status and level of consciousness, ${ }^{7,33,34}$ as well as disease outcome 1year after trauma ${ }^{5,35}$ were assessed according to the clinical scales.

Patients were examined dynamically. At the time of the first study, 25 of them had been diagnosed with different forms of post coma disorders of consciousness (PCDOC) lasting from 2months to 7years, the output of which was characterized by emerging understanding of speech and following instructions consistently). PCDOC was chronic or irreversible in 11 persons, with 5 cases of akinetic mutism and 6 cases of minimal conscious state in the form of inconsistent following single simple instructions (mutism with speech understanding). The rest were in PCDOC lasting up to 7 months, with varying degrees of further mental recovery: from emerging speech (speech disintegration) to light borderline cognitive and emotional-personal disorders.

The control group consisted of 23 healthy subjects aged 18-34(average age 24.3 \pm 5.6 ). The studies were carried out in accordance with the Declaration of Helsinki, after getting informed consent for research from all the subjects and approval by the ethics committee of Institute of Higher Nervous Activity and Neurophysiology (IHNA and NPh) of RAS. While recording EEG, standardized computer tests were run to activate attention: ${ }^{30}$ a red spot appearing, without instruction, against the dark screen at a random moment of time for involuntary attention and a red sphere moving randomly around the screen that a subject, in whatever state, was asked to track with eyes for voluntary attention. EEG orienting response was studied for eye opening, as well as that to frequency-modulated (250, $500,1000 \mathrm{~Hz}$ ) auditory signals with intensity of $60 \mathrm{~dB}$, presented when eyes closed. Behavioral equivalents of different forms of attention were monitored by photographing and filming.

EEG was recorded, according to the international 10-20 system, in 18 channels monopolarly (as oriented to ear indifferent electrodes), in the band $0.3-35 \mathrm{~Hz}$, at resting state, with eyes closed and different types of attention activated. A qualitative evaluation of background EEG patterns and those with afferentation was performed, with further mathematical processing: the spectral-coherent analysis of one-minute artifact less segments and localization of equivalent dipole sources of the single components, using the BrainLoc program. ${ }^{36}$ EEG coherence spectra were calculated in $0.4-\mathrm{Hz}$ increments. EEG coherence (EEG Coh) was calculated for all possible combinations of pairs of cortical sites and the periods of the main biological rhythms (delta, theta, alpha, beta1, beta2) in the band $0.5-20 \mathrm{~Hz}$.

The EEG Coh indicators were calculated as an absolute value of complex spectral coherence squared, averaged over the frequency range, using the Mat Lab 6.0 program, Math Works Inc. Quantitative EEG correlates of AT were calculated, comparing its coherent background characteristics with these in attention states, or comparing those in different states of attention. The statistical analysis of EEGCoh was performed for paired samples that deviate from normal distribution, using the nonparametric Wilcoxon and Mann-Whitney test for bound and unbound samples, respectively. ${ }^{37}$

The electrophysiological data were compared in the norm and pathology, and also those of a general clinical examination of patients, using mathematical statistics. In particular, a correlation analysis of formalized EEG InterCoh reactivity was carried out in 6 cortical regions (Figure 1A) with clinical characteristics of a current state and outcome of traumatic disease: for the entire sample of observations (74 studies in 35 patients with severe TBI and 9 in healthy subjects) (Table 1), including the early stages of consciousness recovery in patients with PCDOC (34 studies in a vegetative state and akinetic mutism) (Figure 2).

Table I Correlation of attention-related EEGInterCoh reactivity with current clinical data in patients with PCDOC $(\mathrm{N}=74)$

\begin{tabular}{lllll}
\hline \multirow{2}{*}{$\begin{array}{l}\text { EEG intercoh } \\
\text { reactivity }\end{array}$} & GSC & \multicolumn{3}{c}{ Consciousness } \\
\cline { 2 - 5 } & SCC & $\mathbf{p}$ & SCC & $\mathbf{p}$ \\
\hline OI-O2 & 0.29 & 0.04 & 0.16 & 0.17 \\
P3-P4 & 0.37 & 0.01 & 0.44 & $<0.001$ \\
C3-C4 & 0.31 & 0.03 & 0.31 & 0.006 \\
F3-F4 & 0.42 & 0.004 & 0.35 & 0.003 \\
FpI-Fp2 & 0.36 & 0.01 & 0.41 & $<0.001$ \\
T3-T4 & 0.43 & 0.002 & 0.31 & 0.008
\end{tabular}

Notes GCS as for Glasgow Coma Scale; consciousness according to Dobrokhotova et al. ${ }^{7}$

Here and in Tables 2-3: SCC is for Spearman's rank correlation coefficient. Greatest values of correlation coefficient are shown in bold

When attention was activated, the changes in EEG InterCoh were compared to the state of the corpus callosum (CC) tracts, according to the data of 3T diffusion tensor imaging (DTI) in 9 healthy subjects and in 25 patients with STBI. For the CC tracts, a differential topographic approach was used. In sagittal sections of the CC, 7 topographic zones were distinguished by 2 independent experts and, thus, the tracts were mapped: splenium, truncus posterior, middle truncus, truncus anterior, genu and rostrum (Figure 1B). ${ }^{38,39}$ Along the tracts of each zone, the fractional anisotropy (FA) was calculated, characterizing the integrity of axons. The correlation coefficient between the measurements by both experts was 0.92 . The topographic correspondence of the mapped $\mathrm{CC}$ zones and EEGInterCoh calculated between the symmetrical occipital, parietal, central, frontal and temporal areas (Figure 1) enabled us to match DTI and EEG parameters. 
A

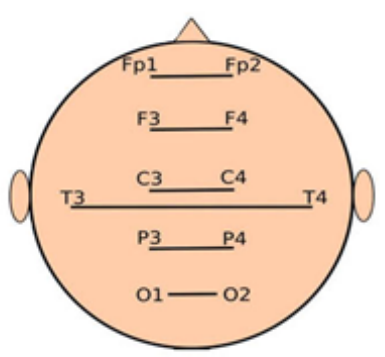

B

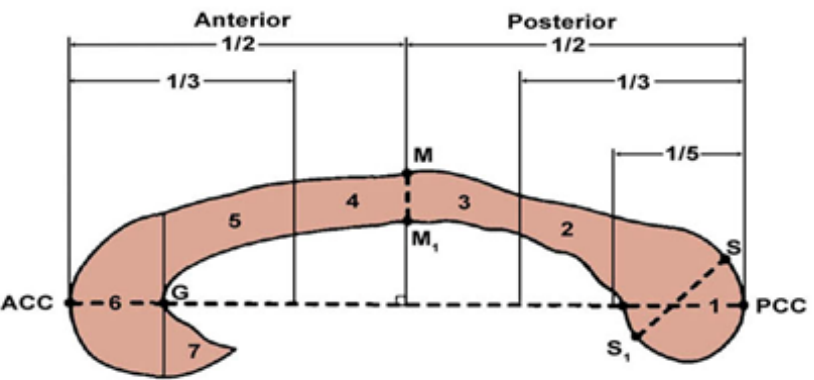

Figure I Regions for assessment of quantitative structural and functional data of EEGInterCoh (A) and CC (B ).

1-splenium, 2-isthmus, 3-truncus posterior, 4-middle truncus and 5-truncus anterior, 6-genu, 7-rostrum.

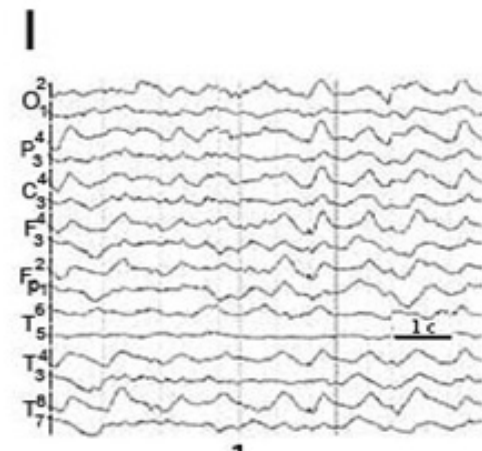

1

II

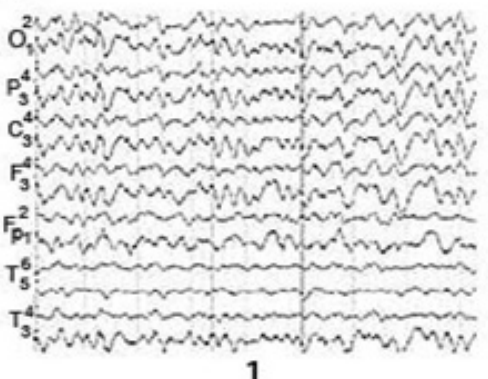

1

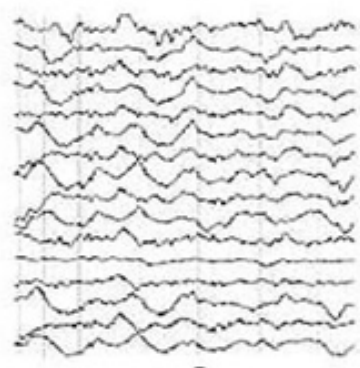

2

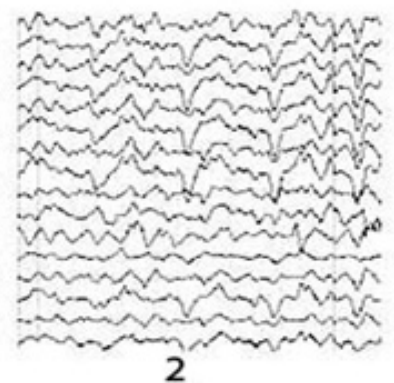

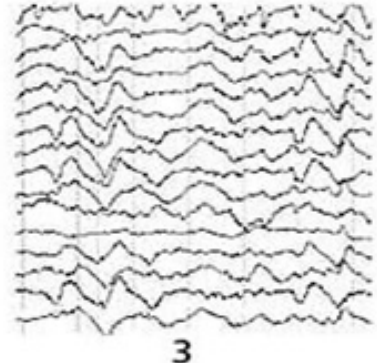

3

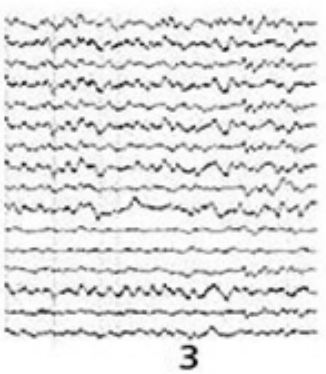

Figure 2 Reactive attention-related changes in EEG pattern in patient Sch. with a chronic PCDOC after STBI in the dynamics.

A. 3months after severe TBI, vegetative state.

B. 4years 3months after severe TBI; akinetic mutism with emotional reactions, following single instructions inconsistently.

I-background; 2- voluntary attention; 3-sound.

When calculating Spearman rank correlation coefficients (SCC) between the current clinical data, those of regional tractography of the CC and EEG reactivity, EEG InterCoh was formalized for each region: 1-no significant changes were observed in any of the analyzed frequency bands, 2-there were significant changes in one or several frequency bands, regardless of their direction.

\section{Results}

Electrographic reactions related to attention activation were detected in almost all the patients with PCDOC in the course of observation. However, the way they manifested (in both EEG pattern and coherence structure) was:

i. significantly different from the norm, ii. variable in time, and

iii. different in form and dynamics in a reversible and chronic forms of PCDOC.

In patients with a chronic PCDOC, attention-related EEG changes were poor and stereotypical both with afferention of different types and over time. The EEG picture showed how a pathologically slow or epileptiform activity of local or paroxysmal type increased in response to given stimuli Figure 2. Emerging groups of fragmental alpha activity in some cases were due to the equivalent dipole sources of the brainstem or the subcortex (hippocampus).

The reactive shifts of EEG Coh occurred concurrently with diffuse decrease inter central connectivity - characteristic for a PCDOC- 
during spontaneous bioelectrical activity in the main frequency bands, as compared to healthy subjects, most pronounced in the frontal cortical areas. ${ }^{40-42}$ In a chronic PCDOC, attention-related changes in EEGCoh prevailed inside hemispheres with the decrease (absence) of interhemispheric reactivity of this parameter, especially in the anterior cortical regions (symmetrical central, frontal and frontal-pole lobes) (Figure 3). They mainly concerned slow (delta and, especially, theta) activity, and often in the form of increasing connectivity in these frequency bands, as compared to the background. In both vegetative state and mutism, EEGCoh changes were observed to be modalitynonspecific Here and in Figure 5: EEGCoh for reaction: greater (red lines)/less (green lines) than in the background $(p<0.05)$ (i.e. their similarity regardless of attention activation method), which indicated a most basic and non-specific form of attention, or orienting response. There were distinguished a few patterns of distinctive EEGCoh changes related to attention activation in a chronic PCDOC. One, more characteristic of a vegetative state, was manifested locally as a connectivity increase in the projection zone of an activated sensor, with insignificant diffuse changes in EEGCoh (Figure 3A). According to literature, this is comparable to brain reactions of newborn babies under 3 months, ${ }^{43,44}$ with the leading role of brain stem commissures in the formation of inter hemispheric connections found in them.

A
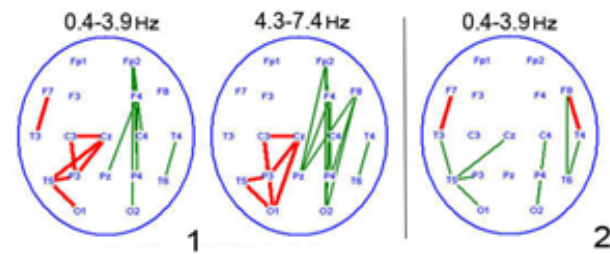

$4.3-7.4 \mathrm{~Hz}$
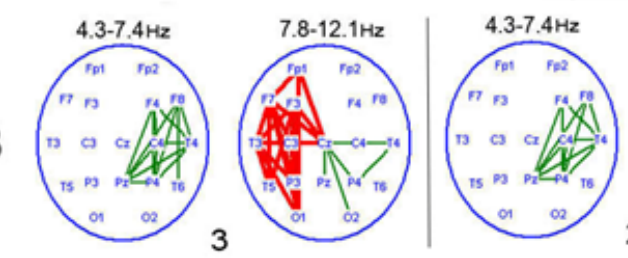

2

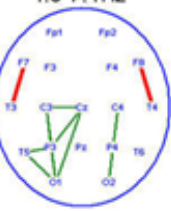

$7.8-12.1 \mathrm{~Hz}$
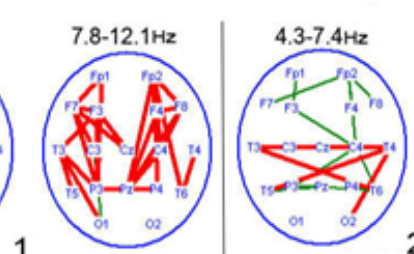

2
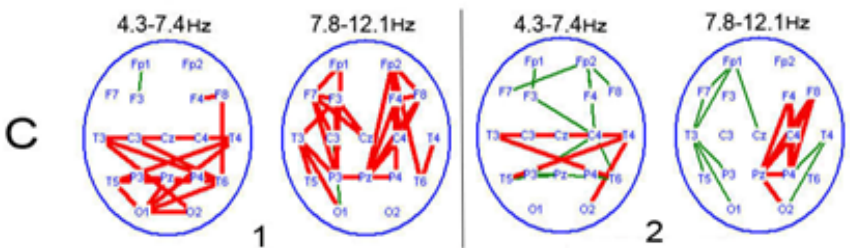

Figure 3 Typical patterns of attention-related EEGCoh changes in patients with a chronic PCDOC.

A. local form: patient Ya., IOmonths after trauma, akinetic mutism with emotional reactions;

B. generalized intra hemispheric form: patient K., 8years after trauma, akinetic mutism with emotional reactions;

C. increase in posterior cortical areas: patient M., 7 years after trauma, akinetic mutism with emotional reactions.

I-opening of the eyes; 2-sound; 3-voluntary attention.

The other pattern was characteristic of akinetic mutism with a predominance of extensive coherence changes within one or both hemispheres and EEG InterCoh unresponsiveness (Figure 3B). Reciprocity between hemispheres was very probable, i.e. an EEGCoh increase in one hemisphere with its decrease in the other. Extensive
EEG reactions, presented mainly by slow (theta) rhythms with little variability in cortical activity (alpha and beta bands), indicated a decrease of cortical and cortico fugal connectivity, with concurrent dominance of the sub cortical structures of the brain. This type of EEGCoh reactivity was considered as a modified orienting response conditioned by the vertical dys connectivity of the stem and cortex.

In patients with different forms of mutism, a reactivity pattern with pronounced changes in symmetrical and diagonal (polysynaptic) EEGInterCoh in the temporo-parieto-occipital region was also observed when attention was activated; it was described in the literature as a characteristic reaction of a 3-6month-old child's brain ${ }^{43,44}$ in the process of interhemispheric integration of the posterior parts of the hemispheres via inter-hippocampal connectivity and neural pathways of the arch. The presence of exogenous attention, driven by external stimuli, may be assumed in those patients..$^{45}$

In patients with a reversible PCDOC, attention-related EEG reactions were more dynamic and variable in different types of stimulation and over time. In the EEG pattern, a reactive increase of local pathological signs in the zone of the most destruction and non-severe signs of median irritation (theta-rhythm bursts) could be observed in the course of one of the studies. With the recovery underway, changes similar to arousal reaction were revealed more often (Figure 4). In the structure of EEGCoh, there was a transition of attention-related dominant reactivity from the theta to alpha band, as consciousness was restored. In addition, in alpha EEG activity, there was observed a switch from its stem-sub cortical sources to the frontobasal (which indicated the involvement of some elements of the fronto-thalamic system in the brain activity), as well as the occipital sources characteristic of the norm.

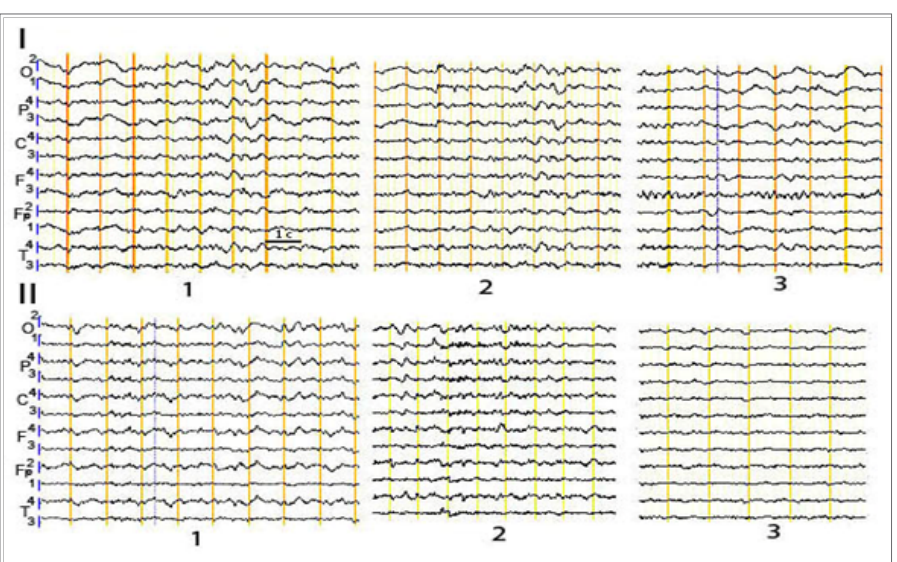

Figure 4 Attention-related changes in EEG pattern in patient $A$. with a reversible PCDOC after STBI in the dynamics.

A. I, 5months after trauma, vegetative state.

B. 2.5 months after trauma, transition from akinetic mutism to following instructions.

I-background; 2-voluntary attention; 3-involuntary attention

The systemic reorganization of EEGCoh with activated attention of patients in a reversible PCDOC was also poorer, compared to the norm: reactively changing (often increasing) coherent connectivity was decreased. However, there was a characteristic interhemispheric reactivity, involving the frontal lobes, already at the early post coma stages (Figure 5). 


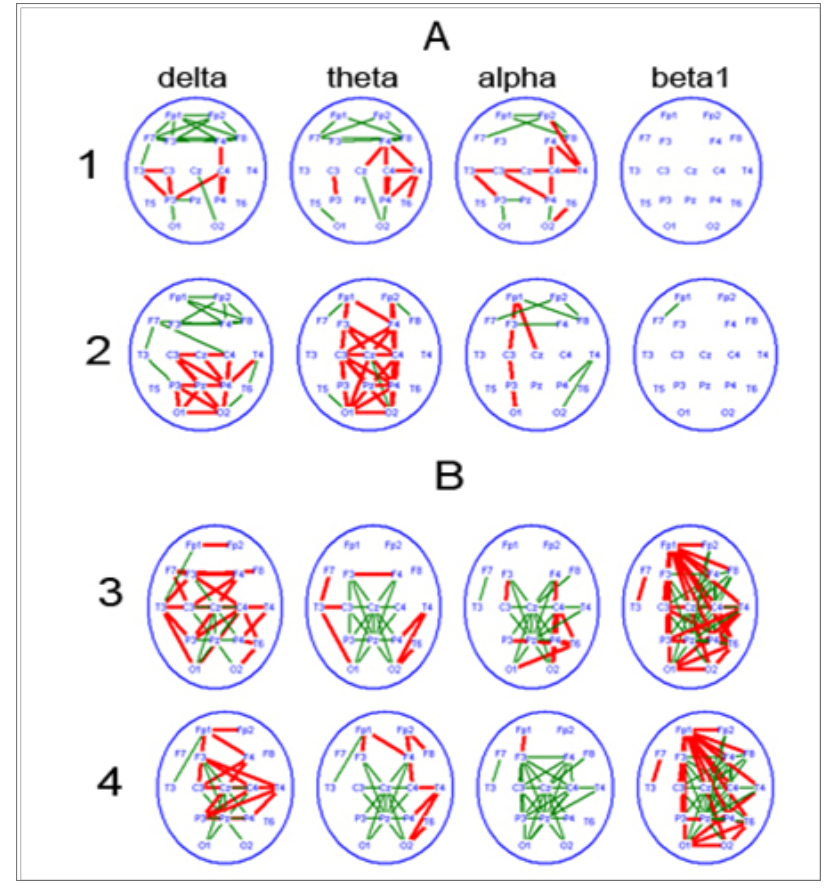

Figure 5 Attention-related changes in EEG coherence over frequency bands of biological rhythms, in patients with a reversible PCDOC and currently in akinetic mutism.

A. Data of patient K. 7months after STBI. Borderline consciousness recovery and mental activity, lyear later.

I-involuntary attention; 2-sound tone.

B. data of the patient I. in 3,5months after STBI. Recovery with severe disability (dementia with disorientation), lyear later.

3-eyes opening; 4-voluntary attention.

Attention-related changes in EEGCoh in the frontal lobes (between symmetric zones, as well as diagonal zones) might reflect the potential of the fronto-thalamic complex, whose involvement is particularly obvious in case of voluntary attention (Figure 5B). In ontogeny, those signs are revealed in children aged aproximately lyear, but the neurophysiological mechanism of attention of a 'frontothalamic type' is completely formed at the age of 7 to $8 .{ }^{46}$

At the early stages of consciousness recovery in patients with a reversible PCDOC, an EEG response to stimuli of different modalities was stereotypical and lateralized, with a predominant EEGCoh increase in the left hemisphere in a vegetative state and in the right one in mutism, indicating the presence of undifferentiated involuntary attention. It was possibly provided by different regulatory regions of the brain, with a stronger role of the brainstem in a vegetative state and of the sub cortex and diencephalon in mutism. ${ }^{31,47}$

We also observed a predominance of modified EEGCoh in the occipital regions in response to visual stimuli and in the temporal regions to auditory ones in some patients, which demonstrated a local component of EEG reactions. As their speech was restored, attentionrelated changes in EEGCoh acquired features of healthy adults. ${ }^{30}$

Correlation analysis showed statistically significant connection of the most clinical and EEGInterCoh signs under consideration. For the entire sample of observations, a correlation with the current functional state values was the strongest in EEGInterCoh reactivity of the symmetrical frontal and parietal cortical zones/regions (Table 1). At the early stages of consciousness recovery-between EEGInterCoh reactivity of the anterior cortical regions (symmetrical central and frontal) and the outcome (Table 2): complete EEGInterCoh Un responsiveness is characteristic only of chronically unconscious patients in a vegetative state or akinetic mutism; in patients recovering to clear consciousness, attention-related EEGInterCoh always changed. The variability of EEGInterCoh of symmetrical temporal regions (Table 1) was low not only in pathology, but also in the norm and thus of little informativeness to distinguish between a chronic and reversible PCDOC.

Table 2 Correlation of attention-related EEGInterCoh reactivity with outcome scales in patients with PCDOC $(\mathrm{N}=34)$

\begin{tabular}{lllll}
\hline \multirow{2}{*}{$\begin{array}{l}\text { EEG intercoh } \\
\text { reactivity }\end{array}$} & GOS & & \multicolumn{2}{c}{ PCDOC reversibility } \\
\cline { 2 - 5 } & $\mathbf{S C C}$ & $\mathbf{p}$ & $\mathbf{S C C}$ & $\mathbf{p}$ \\
\hline OI-O2 & 0.22 & 0.14 & 0.03 & 0.86 \\
P3-P4 & 0.46 & 0.002 & 0.16 & 0.3 \\
C3-C4 & 0.44 & 0.003 & 0.33 & 0.03 \\
F3-F4 & 0.47 & 0.002 & 0.24 & 0.13 \\
FPI-Fp2 & 0.52 & $<0.001$ & 0.31 & 0.04 \\
T3-T4 & 0.33 & 0.03 & 0.11 & 0.47 \\
\hline
\end{tabular}

Notes GOS is for Glasgow Outcome Score ${ }^{35}$

PCDOC reversibility, I-chronic, 2-limitedly reversible, 3-reversible

By qualitative comparisons, a certain connection was found between the nature of EEGInterCoh reactivity and the structural integrity of the $\mathrm{CC}$ that anatomically provided interhemispheric connectivity (Figure 6). The presence of attention-related EEGInterCoh changes in the frontal cortical areas was associated with the relative integrity of the $\mathrm{CC}$ tracts in these regions (the norm and reversible PCDOC); EEGInterCoh unresponsiveness, with generalized (extensive) damage (reduction) of the CC tracts (chronic PCDOC).

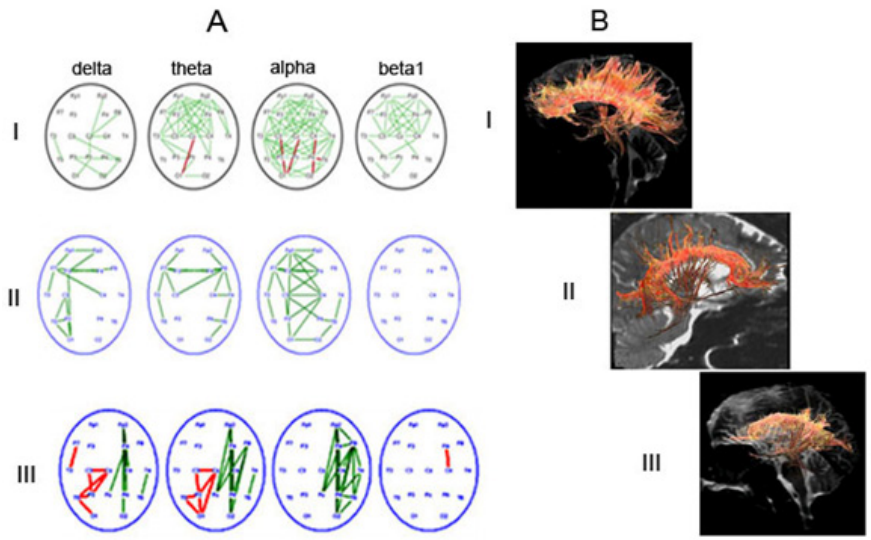

Figure 6 Comparison of characteristic patterns of EEGInterCoh changes, related to eyes opening, and the state of $\mathrm{CC}$ tracts in the norm and in posttraumatic depressed consciousness.

I. The norm $(\mathrm{N}=10$ in $\mathrm{A}$, healthy volunteer in $\mathrm{B})$;

II. Reversible PCDOC (individual observations in A and B,

III. Chronic PCDOC (individual observations in A and B). 
To evaluate the degree of structural determinacy of this EEG parameter, a correlation analysis was carried out for the regional data of attention-related EEGInterCoh reactivity and the fractional anisotropy coefficient of the CC (see Figure 1) (Table 3). As it follows from the data of Table 3, the correlation values between those parameters are highly variable $(0.20-0.68)$, whereas their regional maximum (0.34-0.68) is comparable with clinical data and even exceeds those. There were identified the $\mathrm{CC}$ zones that have the correlation between fractional anisotropy and all the values of EEGInterCoh reactivity non-specifically increased (splenium, genu, rostrum of the $\mathrm{CC}$ ) or decreased (isthmus and truncus posterior). Regionally specific data included strong correlations between EEG reactivity of the symmetrical parietal areas and FA1, and those of interfrontal areas and FA6.

In patients with "irresponsive" EEGInterCoh of those regions, the regional values of fractional anisotropy of the $\mathrm{CC}$ (in the area of genu (F6) and, especially, splenium (F1), respectively) were reduced (less than 0.4), as compared to the "responsive" ones (Figure 7). The nonparametric Mann-Whitney test showed the significance of these differences at the level of $\mathrm{p}<0.001$.

Table 3 Correlation of EEGInterCoh reactivity with regional values of fractional anisotropy of corpus callosum ( $\mathrm{N}=37)$

\begin{tabular}{|c|c|c|c|c|c|c|c|c|c|c|c|c|c|c|}
\hline \multirow{2}{*}{$\begin{array}{l}\text { EEG intercoh } \\
\text { reactivity }\end{array}$} & \multicolumn{2}{|l|}{ FAI } & \multicolumn{2}{|l|}{ FA2 } & \multicolumn{2}{|l|}{ FA3 } & \multicolumn{2}{|l|}{ FA4 } & \multicolumn{2}{|l|}{ FA5 } & \multicolumn{2}{|l|}{ FA6 } & \multicolumn{2}{|l|}{ FA7 } \\
\hline & scC & $\mathbf{p}$ & scC & $\mathbf{p}$ & scC & $\mathbf{p}$ & scC & $\mathbf{p}$ & SCC & $\mathbf{p}$ & scC & $\mathbf{p}$ & scc & $\mathbf{p}$ \\
\hline Ol-O2 & 0.45 & 0.008 & 0.46 & 0.34 & 0.19 & 0.28 & 0.32 & 0.07 & 0.4 & 0.02 & 0.43 & 0.01 & 0.4 & 0.02 \\
\hline P3-P4 & 0.66 & $<0.001$ & 0.43 & 0.01 & 0.33 & 0.06 & 0.45 & 0.01 & 0.42 & 0.02 & 0.53 & 0.002 & 0.54 & 0.01 \\
\hline C3-C4 & 0.34 & 0.05 & 0.2 & 0.25 & 0.31 & 0.08 & 0.27 & 0.12 & 0.13 & 0.48 & 0.29 & 0.09 & 0.32 & 0.06 \\
\hline F3-F4 & 0.49 & 0.004 & 0.3 & 0.1 & 0.35 & 0.05 & 0.42 & 0.02 & 0.44 & 0.01 & 0.59 & $<0.001$ & 0.57 & $<0.001$ \\
\hline Fpl-Fp2 & 0.45 & 0.008 & 0.35 & 0.05 & 0.28 & 0.11 & 0.24 & 0.18 & 0.27 & 0.13 & 0.38 & 0.02 & 0.32 & 0.06 \\
\hline T3-T4 & 0.57 & $<0.001$ & 0.34 & 0.05 & 0.39 & 0.03 & 0.61 & $<0.001$ & 0.55 & 0.001 & 0.67 & $<0.001$ & 0.68 & $<0.001$ \\
\hline
\end{tabular}
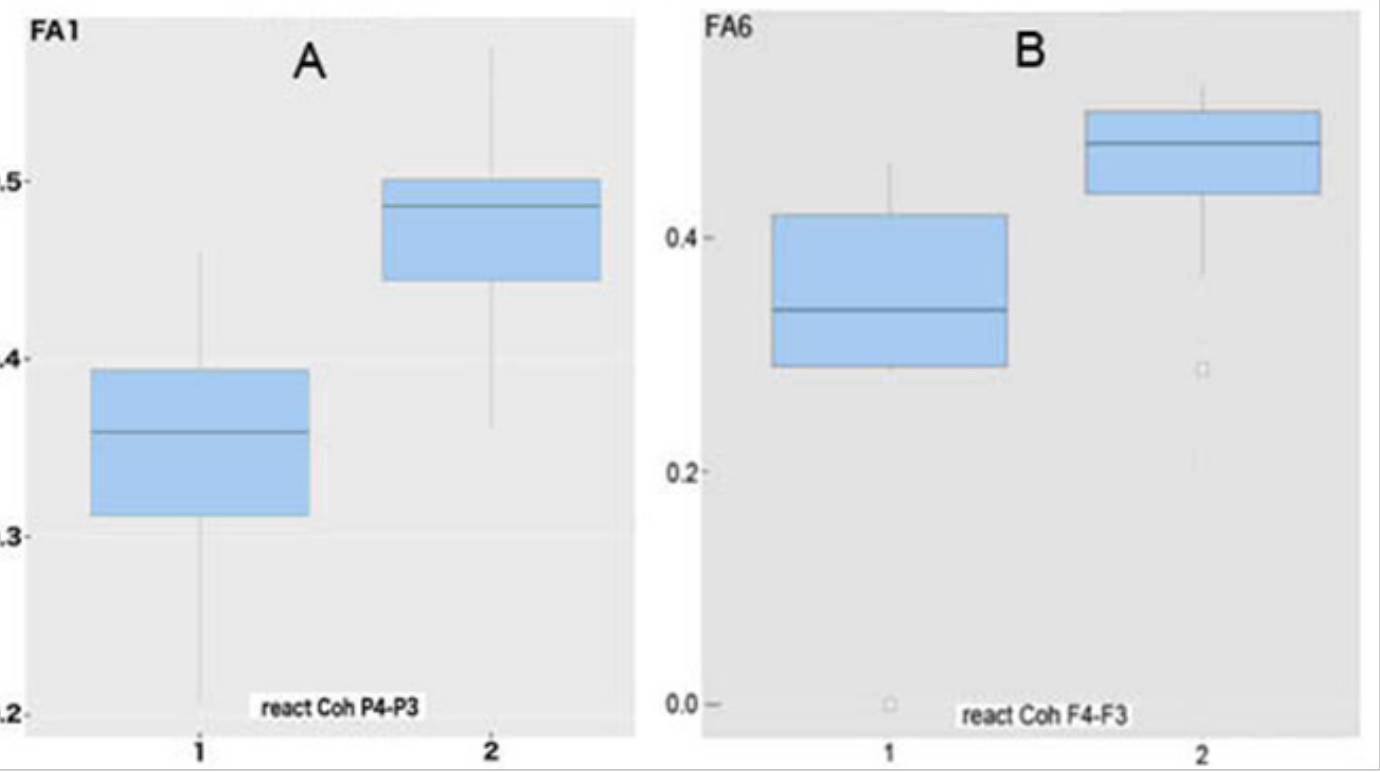

Figure 7 "Boxes with antennae" for fractional anisotropy in area of splenium (A) and genu (B) of corpus callosum in patients with STBI and different reactivity of attention-related EEGInterCoh .

I- Patients with irresponsive attention-related EEGInterCoh of corresponding cortical areas,

2- $\quad$ Responsive. Dark horizontal line in the middle of diagram is median of data sample.

\section{Discussion}

Given how important attention is as one of the basic components of consciousness, ${ }^{2,48-51}$ necessary for its recovery from pathology, ${ }^{52,53}$ a detailed study was conducted on changes in EEG, while activating AT in patients after STBI, at different stages of mental recovery from coma to clear consciousness.
The study results on the presence of changes associated with attention activation, though qualitatively and quantitatively different in a reversible and chronic PCDOC, in EEG of almost all the post coma patients, have a diagnostic and prognostic value. The EEG reactions revealed in the form of intensifying pathological phenomena in response to different stimuli and a local increase in EEGCoh slow rhythms, may reflect the formation of pathological foci in the 
central nervous system with dominant properties described earlier, ${ }^{54}$ which hinder further consciousness recovery. These results raise a complicated problem of suppressing dominant foci. ${ }^{55}$

Prognostically favorable characteristics of a reversible PCDOC included the presence of attention-related changes in EEGInterCoh, and also in the frontal lobes, even at the early stages lacking clear manifestations of consciousness.

This is the first time a significant correlation between the preservation degree of the corpus callosum tracts (primarily, rostrum, genu, splenium) and the attention-related reactivity of EEGInterCoh was revealed, which reflects a certain non-rigid structural determinacy of the latter. In the previously published research, the degree of brain injury was found to correlate significantly only with such integrative parameters as the severity of a condition, the degree of primary depression of consciousness in patients with STBI and its outcome. ${ }^{32,56}$ Structural and functional correlation of attention pathology and disorders of inter- and intra-hemispheric connectivity needs to be further redefined, possibly in other clinical frameworks (stroke, hypoxia or brain tumor).

Our interpretation of EEG results, comprising the published data on the electrographic features of visual perception and the formation of attention function at different ontogenetic stages, ${ }^{29,57}$ is based on the similar dynamics of posttraumatic mental recovery and its formation in ontogenesis. ${ }^{58,59}$ This concept is by far different from that of the similarity of consciousness recovery to awakening..$^{21,60,61}$ However, the latter seems seminal in terms of diagnostics and the further development of neurorehabilitation approaches, with a greater compensatory neuroplasticity of the brain, as compared to gradual awakening. Therefore, the present work demonstrates patients with a chronic PCDOC have prevailing EEG features of involuntary attention, characteristic of infants, which is provided mostly by the limbic system with possible involvement of the parietal cortex. ${ }^{13,14}$ Patients with a reversible PCDOC have, besides EEG manifestations of involuntary AT, a positive indication of the relatively functionally preserved front-thalamic system even at early stages of recovery, which determines the presence (or gradual recovery) of voluntary/ purposeful attention. The degree of structural and functional damage of this system in a post-comatose unconscious state was emphasized to be crucial in the works of S. Laureys et al. ${ }^{62}$ and other authors, ${ }^{63,64}$ based on neuroimaging data. Given the importance of preservation (or recovery) of the structural-functional interhemispheric connectivity, as well as the front-thalamic one, for the formation of attention in a post coma disorders of consciousness, the data obtained may contribute to the development of targeted Neurotherapy. ${ }^{65,66}$

\section{Conclusion}

Thus, the present paper

i. Features attention-related changes in EEG and coherence EEG patterns, characteristic for the early stages of mental recovery (vegetative state, akinetic mutism and emotional reactions).

ii. Reveals qualitatively and quantitatively different reactive changes of interhemispheric coherence EEG in a reversible PCDOC and chronic PCDOC, which are statistically significant and may have a prognostic value, as well as a prognostically favorable changes in interhemispheric coherence EEG associated with attention, including those in the frontal lobes, starting from the early stages lacking any external manifestations of consciousness. iii. Establishes a significant correlation between the preservation degree of the corpus callosum tracts (primarily, rostrum, genu, splenium) and the attention-related reactivity of interhemispheric coherence EEG, which reflects the fact of its being structurally determined, though not rigidly.

\section{Acknowledgements}

None.

\section{Conflict of interest}

The authors declare that the research was conducted in the absence of any commercial or financial relationships that could be construed as a potential conflict of interest.

\section{Funding}

Study was supported by RFFI № 18-013-00967a and № 16-2908255 ofi-M

\section{References}

1. Luria AR. The working brain: An introduction to neuropsychology. New York, NY: Basic Books; 1973. 371 p.

2. Vundt V. Consciousness and attention. In: Leont'ev AN, et al. editors. Anthology of attention. Moscow: Izdatel'stvo Moskovskogo Universiteta; 1976. p. 8-25.

3. Navon D. The importance of being visible: on the role of attention in a mind viewed as an anarchic intelligence system: 2.Application to field of attention. Europ J Cogn Psychol. 1989;1(3):215-238.

4. Potapov AA, Likhterman LB, Kravchuk AD, et al. Traumatic brain injury: problems and prospects. Voprosy neirokhirurgii im NN Burdenko. 2009;2:3-8.

5. Zaitsev OS, Tsarenko SV. Neiroreanimatologiya. The recovery from coma (therapy of postcoma states). Moscow: Litass; 2012. 120 p.

6. Aleksandrova EV, Tenedieva VD, Potapov AA. Post-traumatic unconscious states. Moscow: GEOTAR-Media; 2015. 392 p.

7. Dobrokhotova TA, Potapov AA, Zaitsev OS, et al. Post coma reversible unconscious state. Sotsial'naya I linicheskaya psikhiatriya. 1996;(2):2636.

8. Dobrokhotova TA, Sharova EV, Zaitsev OS, et al. The psychiatric aspect of the problem of adaptation of patients with severe traumatic brain injury undergoing prolonged coma. Voprosy neirokhirurgii im NN Burdenko. 1992;(1):29-32.

9. Monti MM, Coleman MR, Owen AM. Executive functions in the absence of behavior: functional imaging of the minimally conscious state. Prog Brain Res. 2009;177:249-260.

10. Monti MM, Vanhaudenhuyse A, Coleman MR, et al. Willful modulation of brain activity in disorders of consciousness. $N$ Engl $\mathrm{J} \mathrm{Med}$. 2010;36(7):579-89.

11. Sharova EV, Zaitsev OS, Korobkova EV, et al. Analysis of behavioral and EEG correlatives of attention in the dynamics of recovery of consciousness following severe brain injury. Neurology, neuropsychiatry, psychosomatics. 2016;8(3):17-25.

12. Khomskaya ED. Neuropsychology. Moscow: Izdatel'stvo MGU; 2002. p. 225-238.

13. Posner MI, Petersen SE. The attention system of the human brain. Ann Rev Neurosci. 1990;13:25-42 
14. Petersen SE, Posner MI. The Attention System of the Human Brain: 20 Years After. Annu Rev Neurosci. 2012;35:73-89.

15. Machinskaya RI. Neurophysiological mechanisms of voluntary attention (analytical review). Fiziologiya cheloveka. 2003;53(2):133-50.

16. Rizzolatti G, Riggio L, Dascola I, et al. Reorienting attention across the horizontal and vertical meridians: evidebce in favor of a premotor theory of attention. Neuropsychology. 1987;25(1A):31-40.

17. Bratus' BS, editor. General psychology. Vol. 4. Attention. Moscow: Akademiya; 2006. p. 248-251.

18. Naatanen P. Vnimanie i funktsii mozga [Attention and brain function]. Moscow: Izdatel'stvo Moskovskogo universiteta; 1998. 556 p.

19. Salil HP, Pierre NA. Characterization of N200 and P300: selected studies of the event-related potential. Int J Med Sci. 2005;2(4):147-154.

20. Oknina LB, Sharova EV, Zaitsev OS, et al. Long-latency components of acoustic evoked potentials (N100, N200 and P300) to predict recovery of consciousness in patients with severe traumatic brain injury. Voprosy neirokhirurgii im N N Burdenko. 2011;(3):19-30.

21. Gnezditskii VV, Piradov MA. Neurophysiology of coma and disorder of consciousness (analysis and interpretation of clinical observations). Ivanovo: Press Sto; 2015. 528 p.

22. Moruzzi G, Magoun HW. Brain stem reticular formation and activation of EEG. EEG and Clin Neurophysiol. 1949;1(4):455-73.

23. Sokolov EN. The nervous model of a stimulus and the orienting reflex. Vopr Psikhol. 1960;4:128-137.

24. Machinskii NO, Machinskaya RI, Trush VD. Electrophysiological study of the functional organization of the human brain at the direction of the attention. Message 1.Normal adults. Fiziologiya cheloveka. 1990;16(2):515 .

25. Danilova NN, Astaf'ev SV. The attention of the person as a specific relationship of EEG rhythms with wave modulators of the cardiac rhythm. Zhurnal vysshei nervnoi deyatel'nosti. 2000;50(5):791-803.

26. Knyazeva MG, Fornari E, Vtuli R, et al. Imaging of a synchronous neuronal assembly in the human visual brain. Neuro Image. 2006;29(2):593-604.

27. Stroganova TA, Posikera IN, Pisarevskii MV, et al. EEG $\theta$ rhythm in preterm and full-term infants at the age of five months in endogenous attention. Human Physiology. 2006;32(5):517-527.

28. Fourcher JR, Otzenberg H, Gounot D. Where arousal meets attention: a simultaneous fMRI and EEG recording study. Neuro Image. 2004;22(2):688-697.

29. Farber DA, Beteleva TG. Formation of the system of visual perception in ontogeny. Human Physiology. 2005;31(5): 515-524

30. Sharova EV, Boldyreva GN, Kulikov MA, et al. EEG correlates of the states of visual and auditory attention in healthy subjects. Human Physiology. 2009;35(1):1-10.

31. Boldyreva GN, Sharova EV, Dobronravova IS. The role of cerebral regulatory structures in the formation of human EEG. Hum Physiol. 2000;26( 5):523-538.

32. Zakharova N, Kornienko V, Potapov A, et al. Neuroiaging of traumatic brain injury. London: Springer; 2014. 159 p.

33. Teasdale G, Jennet B. Assessment of coma and impaired consciousness. A practical scale. Lancet. 1974;2(7872):81-44.

34. Dobrokhotova TA, Grindel' OM, Bragina NN, et al. Recovery of consciousness after prolonged coma in patients with severe traumatic brain injury. Zhurnal nevropatologii $i$ psikhiatrii im SS Korsakova. 1985;85(5):720-726.
35. Jennet B., Bond M. Assessment of outcome after severe brain damage: A practical scale. Lancet. 1975;1(7905):480-484.

36. Koptelov YuM, Gnezditskii VV. Analysis of «scalp potential fields» and three-dimensional localization of sources of epileptic activity of the human brain. Zhurnal nevropatologii i psikhiatrii im SS Korsakova. 1989;89(6):11-8.

37. Voronov VG, Shchekut'ev GA, Grindel' OM. A software package for statistical comparison of EEG traces. Clinical neuroscience: neurophysiology,neurology, neurosurgery. Proceedings of the international conference. Gurzuf; 2003. p. 22-24.

38. Witelson, Sandra F. Hand and sex differences in the isthmus and genu of the human corpus callosum. Brain. 1989;112(Pt 3):799-835.

39. Tkachenko LA, Toronova NO, Krasnoshchekova EI, et al. Comparative MRI morphometric study of the corpus callosum at term and preterm infants. Human Physiology. 2014;40(1):29-34.

40. Sharova EV, Kulikov MA, Zaitsev OS. The peculiarities of EEG dynamics during mental recovery after long-term posttraumatic coma. EEG and Clinical Neurophysiology. 1997;103(1):207.

41. Grindel' OM, Romanova NV, Zaytsev OS, et al. Mathematical analysis of EEG in consciousness recovery after traumatic traumatic brain injuries. Zh nevrol psikhiatr. 2006;106(12):47-51.

42. Boldyreva GN, Zhavoronkova LA, Sharova EV, et al. Electroencephalografic intercentral interaction as a reflection of normal and pathological human brain activity. The Spanish Journal of Psychology. 2007;10(1):169-179.

43.

44. Farber DA, Dubrovinskaya NV, Functional organization of developing brain: age related features and certain regularities. Fiziologiya cheloveka. $1991 ; 17(5) ; 17$.

45. Semenovich AV. Formation of interhemispheric interaction in ontogenesis - in book. Inter hemispheric interaction (edited by AV Semenovich, MS Kovyazina) M: Genesis; 2009. 400 p.

46. Posner MI, Raichle ME. Images of mind. New York: Scientific American Library; 1994.

47. Machinskaya RI. Functional maturation of the brain and formation of the neurophysiological mechanisms of selective voluntary attention in young schoolchildren. Human Physiology. 2006;32(1):20-29.

48. Bragina NN, Dobrokhotova TA. Functional human asymmetry. M: Medicine; 1988, $221 \mathrm{p}$.

49. Dehaene S, Changeux JP. Experimental and theoretical approaches to conscious processing. Neuron. 2011;70(2):200-227.

50. Bor D, Seth AK. Consciousness and the prefrontal parietal network: insights from attention, working memory, and chunking. Front Psychol. 2012;3:63.

51. Bergström F. Eriksson J. Maintenance of non-consciously presented information engages the prefrontal cortex. Front Hum Neurosci. 2014;8:1-10.

52. Raffone A, Narayanan Srinivasan N, Cees van Leeuwen C. The interplay of attention and consciousness in visual search, attentional blink and working memory consolidation. Philos Trans $R$ Soc Lond B Biol Sci. 2014;369(1641):20130215.

53. Monti MM, Pickard JD, Owen AM. Visual cognition in disorders of consciousness: From V1 to top-down attention. Human Brain Mapping. 2013;34(6):1245-1253.

54. Srivas Chennu S, Bekinschtein TA. Arousal modulates auditory attention and awareness: insights from sleep, sedation, and disorders of consciousness. Front Psychol. 2012;3:65. 
55. Sharova EV. Electrographic correlates of brain reactions to afferent stimuli in postcomatose unconscious states after severe brain injury. Human Physiology. 2005;31(3):245-254.

56. Naryshkin AG, Gorelik AL, Skoromets TA, et al. The possibilities for partial rehabilitation of the integrative activity of the brain in patients in a vegetative state. Human Physiology. 2014;40(5):532-541.

57. Potapov AA, Zakharova NE, Kornienko VN, et al. The neuroanatomical foundation for traumatic coma: clinical and magnetic resonance correlates. NN Burdenko J Neurosurg. 2014;(1):4-12.

58. Sergienko EA. The problem of perception of action and representation in the early ontogenesis of man. Development of manual actions in infancy -in Studies on cognitive psychology (Ed. E.A. Sergienko). M: Publishing house "Institute of Psychology RAS"; 2004. p. 227-294.

59. Myagi MA. Long-lasting unconscious state. Auto ref diss cand med sci, Tartu; 1969. 25 p.

60. Dobrokotova TA, Sharova EV, Zaitsev OS, et al. Psychiatric aspect of the problem of adaptation of patients with severe craniocerebral trauma, who have suffered prolonged coma. Voprosy neirokhirurgii im NN Burdenko. 1992;1:29-32.

61. Laureus S, Tononi G. The Neurology of Consciousness. London: Elssevier; 2009. 423 p.
62. Monti MM, Sannita WG. Brain Function and Responsiveness in Disorders of Consciousness. Switzerland: Springer International Publishing; 2016.

63. Laureys S, Faymonville ME, Luxen A, et al. Restoration of thalamocortical connectivity after recovery from persistent vegetative state. Lancet. 2000;355(9217):1790-1791.

64. Lutkenhoff ES, Chiang J, Tshibanda LE. et al. Thalamic and extrathalamic mechanisms of consciousness after severe brain injury. Ann Neurol. 2015;78(1):68-76.

65. Monti MM, Rosenberg M, Finoia P, et al. Thalamo-frontal connectivity mediates top-down cognitive functions in disorders of consciousness. Neurology. 2015;84(2):167-173.

66. Kopachka M, Troshina E, Sharova E, et al. Oknina Diagnostic and therapeutic possibilities of transcranial magnetic stimulation in patients after traumatic brain injury. Accepted abstracts of ECCN 2017. Clinical Neurophysiology. 2017;128(9):e249-e250.

67. Pannasch S, Helmert JR, Müller R, Velichkovsky BM. The analysis of eye movements in the context of cognitive technical systems: Three critical issues. Lecture Notes in Computer Science. 2012;7403:19-34. 